\title{
Twin Gestation with Complete Hydatidiform Mole and Demise of Co-Existing Fetus
}

\author{
Ravikanth Reddy
}
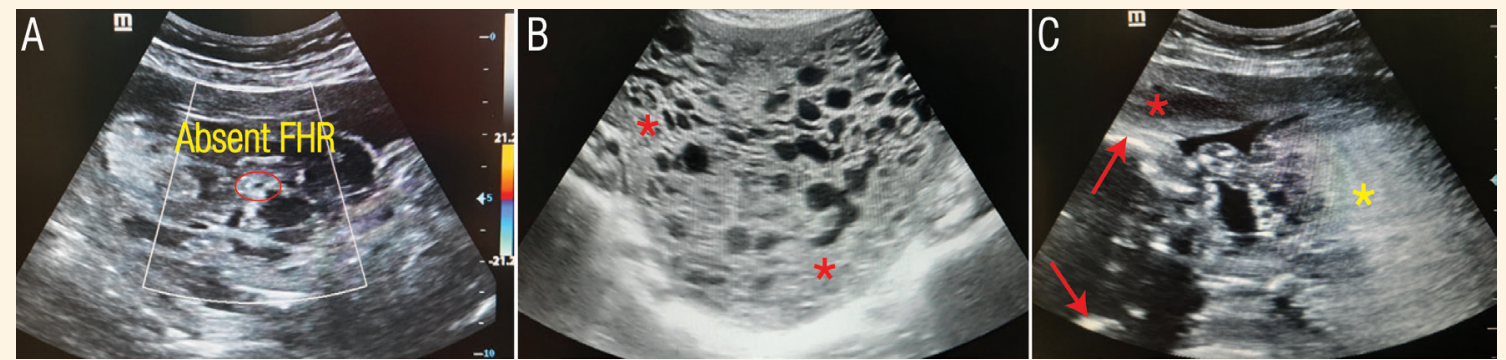

Figure 1: A: Color Doppler image showing fetus with absent fetal heart rate suggesting intrauterine fetal demise. B: High-resolution transverse ultrasonography image demonstrating intrauterine echogenic mass (asterisks) with honeycomb like pattern and absent fetal parts suggestive of a complete hydatidiform mole. C: High-resolution longitudinal ultrasonography image demonstrating fetal head (arrows) situated in the region of uterine fundus whereas the molar mass (yellow asterisk) is noted adjacent to the region of cervical os in the lower uterine segment. The anteriorly situated placenta (red asterisk) can be seen separately.

A

26-YEAR-OLD PRIMIGRAVIDA AT 20 WEEKS of gestation was admitted to the Obstetrics and Gynecology Department, Bengaluru, India, in 2021 with complaints of severe lower abdominal pain and bleeding per vaginum for one day. The patient had vaginal spotting one week prior to the date of presentation with the passage of grape like vesicles. At the time of presentation, the uterus was large for a date corresponding to 28 weeks of gestation (20 weeks on ultrasonography) with additional clinical features such as excessive retching suggesting hyperemesis gravidarum. The patient was hemodynamically stable with no clinical features to suggest distress. Vitals such as pulse, blood pressure and respiratory rate were within normal limits. Coagulation profile and thyroid function tests returned normal results. Moreover, the patient tested negative for toxoplasmosis, rubella cytomegalovirus, herpes simplex and HIV (TORCH) screening profile.

The ongoing bleeding characteristics included the passage of dark brown to bright red blood accompanied by clots and admixed with passage of grape-like cysts per vaginum. However, establishing a final diagnosis from the characteristics of the bleeding alone was difficult in this case, as the molar pregnancy characteristics such as bleeding and lower abdominal pain were indistinguishable from those of a miscarriage. On palpation, the uterine size corresponded to 20 weeks of gestational age. An urgent transabdominal (TAS) obstetric ultrasonography scan was requested which revealed a twin pregnancy with a normal fetus and an echogenic mass with honeycomb like pattern and absent fetal parts. However, the normal fetus at the time of ultrasonography did not demonstrate spontaneous fetal movements and fetal heart rate [Figure 1A].

The demised fetus was situated in the region of uterine fundus whereas the echogenic mass which was suggestive of a complete hydatidiform mole was situated adjacent to the region of cervical os in the lower uterine segment [Figures 1B \& C]. Dimensions of the dead fetus were $9.3 \times 6.9 \times 10.2 \mathrm{~cm}$ with gestational age corresponding to 20 weeks. Serum ß-HCG (Beta human chorionic gonadotrophin) levels of the patient were found elevated at 217,341 mIU/mL. Chest radiograph did not reveal any focal abnormality. The patient along with her husband were counselled regarding the implications of molar pregnancy and the couple were informed regarding risks involved with the progression along the spectrum of gestational trophoblastic disease into more serious entities such as invasive mole and gestational choriocarcinoma, following which the couple had opted for a conservative management approach. On day one of admission, subsequent evacuation of the uterine cavity was performed via the vaginal route which revealed a 




Figure 2: Histopathology image using haematoxylin and eosin staining at $\times 400$ magnification of the evacuated specimen demonstrating trophoblastic proliferation and hydropic degeneration of villi consistent with features of a hydatidiform mole.

dead fetus and vesicular tissue of the molar pregnancy. Post-procedural recovery period was uneventful and weekly measurements of $\beta$-HCG were performed during the post-evacuation follow-up. The presence of a dead fetus was histopathologically confirmed with a co-existing complete hydatidiform mole and a normal placental tissue [Figure 2]. Follow-up at 3 months revealed normal serum $B$-HCG levels without requiring therapy with cytotoxic agents. At 12 months follow-up, serum 3 -HCG levels remained normal without evidence of persistent trophoblastic disease.

The authors certify that they have obtained all appropriate patient consent forms. In the form, the patients have given their consent for their images and other clinical information to be published.

\section{Comment}

A normal fetus in a twin pregnancy with co-existing complete hydatidiform mole is an extremely rare entity, with a worldwide incidence ranging from one in 22,000 to one in 100,000 pregnancies. ${ }^{1}$ The condition poses major challenges during expectant management due to complications such as early fetal demise, bleeding per vaginum, pre-eclampsia, intrauterine fetal growth restriction, hyperthyroidism and torsion of theca lutein cysts. Twin pregnancy with concomitant occurrence of live fetus and coexisting complete hydatidiform mole is also known as 'sad fetus syndrome', due to the coexisting fetus is potentially viable and usually has normal chromosomes. ${ }^{2}$ However, in the current case scenario, there was fetal demise at 20 weeks of gestation due to haemorrhage, which is a known and potentially fatal complication associated with this condition.

Zilberman et al. performed a systematic review and meta-analysis on 14 studies having 244 twin pregnancies with healthy co-twins and coexisting complete hydatidiform moles to ascertain the risk of continuing the pregnancy; ${ }^{3}$ it interpreted that there was an $80 \%$ incidence of maternal complications in ongoing pregnancies-including vaginal bleeding, hyperthyroidism and pre-eclampsia, a $50 \%$ chance of a live birth and a $34 \%$ chance of persistent gestational trophoblastic disease development-subsequently on continuation of pregnancy. However, termination of the pregnancy should only be considered if the patient has significant vaginal bleeding or develops severe pre-eclampsia or thyrotoxicosis or when embolisation of gestational trophoblastic tissue is suspected. Nevertheless, close monitoring and timely surveillance regarding the patient's well-being is recommended for identifying suspicious signs relating to maternal and fetal complications. A conservative approach may be deemed possible with the availability of resources and high standard of care. ${ }^{4}$ A literature review also recommended preoperative placement of bilateral uterine artery balloon catheters to minimise blood loss from the molar pregnancy during the process of delivering healthy and live coexisting twins as torrential hemorrhage is an expected complication of this rare entity. ${ }^{5}$

Comprehensive counselling regarding the risks and management approach, long-term follow-up and timely intervention are imperative for the expectant management of this rare yet clinically relevant presentation of complete hydatidiform mole and coexisting twin gestation.

\section{ACKNOWLEDGEMENT}

The author wishes to thank his assistants, Ms. Sweta and Ms. Babika, for help rendered in retrieving ultrasonography images.

\section{References}

1. Liu Y, Zheng X, Wang Y, Li Y, Liu C. Identification of a hydatidiform mole in twin pregnancy following assisted reproduction. J Assist Reprod Genet 2020; 37:603-10. https:// doi.org/10.1007/s10815-019-01650-3.

2. Rajasekaran K, Dadhwal V, Jassim M. Incidental diagnosis of sad fetus syndrome in triplets. BMJ Case Reports CP 2021; 14:e238977. https://doi.org/10.1136/bcr-2020-238977.

3. Zilberman Sharon N, Maymon R, Melcer Y, Jauniaux E. Obstetric outcomes of twin pregnancies presenting with a complete hydatidiform mole and coexistent normal fetus: A systematic review and meta-analysis. BJOG 2020; 127:1450-7. https://doi.org/10.1111/1471-0528.16283.

4. Sheik S, Al-Riyami N, Mathew NR, Al-Sukaiti R, Qureshi A, Mathew M. Twin Pregnancy with a Complete Hydatidiform Mole and a Coexisting Live Fetus: Rare entity. Sultan Qaboos Univ Med J 2015; 15:e550-3. https://doi.org/10.18295/squmj.2 015.15.04.019.

5. Johnson C, Davitt C, Harrison R, Cruz M. Expectant Management of a Twin Pregnancy with Complete Hydatidiform Mole and Coexistent Normal Fetus. Case Rep Obstet Gynecol 2019; 2019:8737080. https://doi.org/10.1155/2019/8737080. 